\title{
Der Lizenzierungsweg Fernstudium in der Sozialen Arbeit: Der Boom des großen Unbekannten
}

\author{
Nikolaus Meyer • Christina Buschle
}

Eingegangen: 9. März 2020 / Angenommen: 7. September 2020 / Online publiziert: 3. November 2020 (C) Der/die Autor(en) 2020

Zusammenfassung Eine Möglichkeit in das Berufsfeld der Sozialen Arbeit einzumünden besteht im Besuch eines Fernstudiengangs. Der vorliegende Beitrag beleuchtet die Entwicklung der Studierendenzahlen bei Fernstudiengängen aus dem Bereich der Sozialen Arbeit. Dafür werden sowohl Ergebnisse des Statistischen Bundesamtes als auch aus einer Sekundäranalyse zu Einstellungen von Fernstudierenden der Sozialen Arbeit abgebildet. Denn trotz steigender Bedeutung für die Professionalisierung des Berufsfeldes, gemessen an den Studierendenzahlen, ist das Fernstudium Sozialer Arbeit bisher kaum Gegenstand empirischer Analysen. Deutlich wird, dass das Fernstudium für den Lizenzierungsprozess eine hohe Bedeutung hat und ebenso als Möglichkeit der individuellen Professionalisierung für die Beschäftigten.

Schlüsselwörter Professionalisierung · Soziale Arbeit · Hochschulforschung · Fernstudium $\cdot$ Lizenzierung

Prof. Dr. N. Meyer $(\bowtie)$

Fachbereich Sozialwesen, Hochschule Fulda, Leipziger Straße 123, 36037 Fulda, Deutschland

E-Mail: nikolaus.meyer@sw.hs-fulda.de

Prof. Dr. C. Buschle

Arbeitsbereich Erwachsenenpädagogik, IUBH Internationale Hochschule, Kaiserplatz 1, 83435 Bad

Reichenhall, Deutschland

E-Mail: c.buschle@iubh-fernstudium.de 


\section{The licensing path distance learning in social work: the boom of the great unknown}

Abstract Attending a distance learning course is one way of entering the professional field of social work. This article examines the development of student numbers in such distance learning courses. We discuss data from the Federal Statistical Office as well as findings from a secondary analysis of attitudes of distance learning students in social work. So far, and despite its increasing importance for the professionalisation of the field, this alternative path has hardly been subject of empirical analyses. Our findings emphasise the significance of distance learning for the process of licensing as well as for individual professionalisation of employees.

Keywords Professionalization · Social work · University research · Distance learning $\cdot$ Licensing

\section{Einleitung}

Die berufssoziologischen Arbeiten von Everett Hughes (1984) markieren mit den Begriffen „Lizenz“ und „Mandat“ zwei relevante Kategorien zum Verständnis von Berufsgruppen (vgl. Nittel 2000; Schütze 1992). Während nun das Mandat den gesellschaftlichen Auftrag ausdrückt und für die spätere Berufspraxis zentral ist, werden die Noviz*innen in jeder Berufsgruppe an einem zentralen Ort in die konkreten Praktiken der spezifischen Berufsgruppe ,eingeweiht“. Hier erwerben die angehenden Fachkräfte die formale Berechtigung (Lizenz) zum Vollzug auch riskanter Handlungen an ihren Adressat*innen (Nittel 2000; Schütze 1992). Zentrale Orte dieses Lizenzierungsprozesses sind in der Sozialen Arbeit ${ }^{1}$ in besonderer Weise Hochschulen für angewandte Wissenschaft und Universitäten. Hier finden allerdings aktuell erhebliche Wandlungsprozesse statt (vgl. Meyer 2020, 2019a; Meyer und Schoneville 2020; Autorengruppe Fachkräftebarometer 2019). Zunächst auf institutioneller Ebene: Hier engagieren sich zunehmend private Hochschulen und dabei nehmen gerade die Zahlen dualer Studierender erheblich $\mathrm{zu}^{2}$. Gerade letztere Entwicklung wird in Profession und Disziplin Sozialer Arbeit sehr kritisch begleitet (Deutsche Gesellschaft für Soziale Arbeit (DGSA) 2019), kontrovers diskutiert (Otto 2018) und ist zunehmend Gegenstand eigenständiger Forschung (DGSA 2018; Meyer 2018). Trotz der eher geringen Zahl von rund 5\% der Studierenden im Verhältnis zu den Gesamtstudierenden, wird dem dualen Studium sozialer Berufe also eine erhebliche Aufmerksamkeit zu teil. Dabei geraten quantitativ zentrale

\footnotetext{
1 Der vorliegende Beitrag folgt zwei Vorgaben: 1.) Unter dem Begriff „Soziale Arbeit“ werden alle Studiengänge subsumiert, die von Destatis als Sozialwesen (Soziale Arbeit, Sozialpädagogik und Sozialwesen) sowie Pädagogik der frühen Kindheit ausgewiesen werden (vgl. Thole 2014). 2.) Der Beitrag orientiert sich an der Fächerklassifikation des Statistischen Bundesamtes (2018a, b). Zur Kritik an dieser mindestens intransparenten Differenzierung durch das Statistische Bundesamt: Meyer 2019b.

2 Im Wintersemester 2018/2019 wurden 4828 dual Studierende in den untersuchten Studiengängen von Destatis erfasst (Meyer 2020). Im Verhältnis zur Gesamtstudierendenzahl sind dies $5 \%$ der immatrikulierten Personen.
} 
Veränderungen bisher nur geringfügig in den Blick von Profession und Disziplin: Fernstudiengänge expandieren in nicht gekannter Weise. Bereits heute gibt es mehr Fernstudierende als Personen, die ihr Studium dual organisiert haben. Das Fernstudium als Organisationsmodus des Studiums und die Folgen für Professionalisierung sowie Professionalität werden dabei empirisch in der Sozialen Arbeit kaum untersucht. Er wird stattdessen vielmehr als didaktisches Problem reflektiert (vgl. Arnold et al. 2018).

Vor diesem Hintergrund beschreibt der vorliegende Beitrag auf Basis einer Sonderauswertung von Daten des Statistischen Bundesamtes (Destatis) die Entwicklung der Studierendenzahlen in den verschiedenen Fernstudiengängen der Sozialen Arbeit. Darüber hinaus werden die Studierenden in Fernstudiengängen Sozialer Arbeit im Hinblick auf soziodemografische Faktoren sowie deren Erwartungen und Wünschen an das Studium auf Basis der Trendstudie Fernstudium der IUBH (Sommerfeld 2019) in den Blick genommen. Letztere ist dabei aktuell die einzige Quelle zur Studierendenstruktur sowie deren Erwartungen in Fernstudiengängen der Sozialen Arbeit, was angesichts der zunehmenden Bedeutung der Lizenzierungsphase überrascht. Gerade auch, weil die verschiedenen Hochschulen ein Fernstudium höchst unterschiedlich organisieren: Hier existieren Blended Learning-Szenarien neben reinen Onlinestudiengängen oder eher berufsbegleitenden Formen der Qualifizierung für die Soziale Arbeit.

\section{Das Fernstudium in der Fächergruppe Soziale Arbeit}

Insgesamt haben im zuletzt durch Destatis ${ }^{3}$ ausgewiesenen Wintersemester 2018/2019 96.473 Personen einen Studiengang Soziale Arbeit (59.132), Sozialpädagogik (9052), Sozialwesen (24.875) oder Pädagogik der frühen Kindheit (3414) studiert (Meyer 2020).

Im Verhältnis zu der Gesamtstudierendenzahl in den untersuchten Studiengängen studieren 10,6\% (10.261) der immatrikulierten Personen im Fernstudium ${ }^{4}$. Dies unterscheidet sich je nach Studiengang: So studierten 7459 Personen (davon $78 \%$ Frauen) im Wintersemester 2018/2019 einen Fernstudiengang der Sozialen Arbeit, 1101 (davon 81,7\% Frauen) ein Fernstudium im Bereich Sozialpädagogik, 1009 (davon $71 \%$ Frauen) einen entsprechenden Studiengang Sozialwesen sowie 692 Personen (davon $95 \%$ Frauen) einen Fernstudiengang der Pädagogik der frühen Kindheit.

In Abb. 1 zeigt sich der enorme Zuwachs in absoluten Zahlen innerhalb von rund zehn Jahren: Diese entsprechen im Fernstudiengang Soziale Arbeit einer prozentualen Zunahme von über $1047 \%$ bei den Gesamtfernstudierendenzahlen zwischen 2007/2008 und 2018/2019, im Fernstudiengang Sozialpädagogik sogar um $100.800 \%$ (sic!), im Fernstudiengang Sozialwesen um über $128 \%$ sowie im Fernstudium der Pädagogik der frühen Kindheit um über $69.100 \%$.

\footnotetext{
3 Genauere Informationen zum methodischen Vorgehen liefert der Qualitätsbericht des Statistischen Bundesamtes „Studierende an Hochschulen“ (2019).

4 Eine Differenzierung nach den Abschlussarten der Fernstudierenden (Bachelor und Master) kann nicht auf der Grundlage der vorliegenden Destatis-Datensätze nicht getroffen werden.
} 


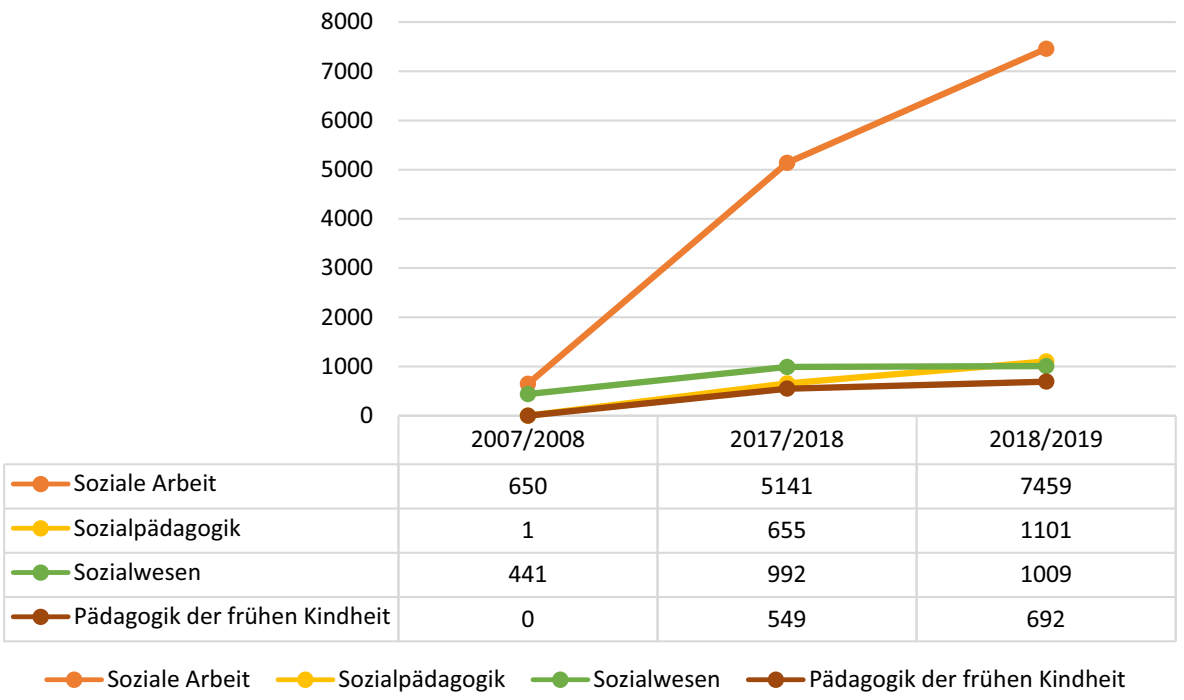

Abb. 1 Entwicklung der Gesamtstudierendenzahlen in den Fernstudiengängen Soziale Arbeit, Sozialpädagogik, Sozialwesen und Pädagogik der frühen Kindheit in der Bundesrepublik Deutschland zwischen 2007/2008 und 2018/2019 (eigene Darstellung, in absoluten Zahlen)

Auch die Bedeutungszunahme der privaten Hochschulen für angewandte Wissenschaften (HAW) im Fernstudium wird deutlich. Grundsätzlich ergänzen Hochschulen in privater oder auch kirchlicher Trägerschaft den öffentlichen Hochschulbereich. Diese Lage hat sich zwischenzeitlich völlig verändert (Abb. 2). Private Hochschulen für angewandte Wissenschaft sind im Segment des Fernstudiums keineswegs mehr eine Ergänzung der Hochschullandschaft, vielmehr dominieren sie diesen Bereich quantitativ deutlich:

- An staatlichen Hochschulen angewandter Wissenschaft studieren insgesamt 2908 Personen einen der genannten Studiengänge.

- An kirchlichen Hochschulen studieren 405 Personen einen entsprechenden Fernstudiengang.

- An privaten HAWs studieren dagegen insgesamt 6948 Personen einen Fernstudiengang.

Ein Vergleich der prozentualen Anteile der verschiedenen Träger (Abb. 2) an den unterschiedlichen Studiengängen offenbart das deutliche Gewicht privater Hochschulen:

- Im Fernstudiengang Soziale Arbeit studieren $21 \%$ der Studierenden an einer staatlichen, 5,2\% an einer kirchlichen sowie $74 \%$ an einer privaten HAW.

- Im Fernstudiengang Sozialpädagogik studieren 7\% der Studierenden an einer staatlichen sowie $93 \%$ an einer privaten HAW (vgl. Meyer 2019b).

- Im Fernstudiengang Sozialwesen studieren $98 \%$ der Studierenden an einer staatlichen sowie $2 \%$ an einer kirchlichen HAW. 


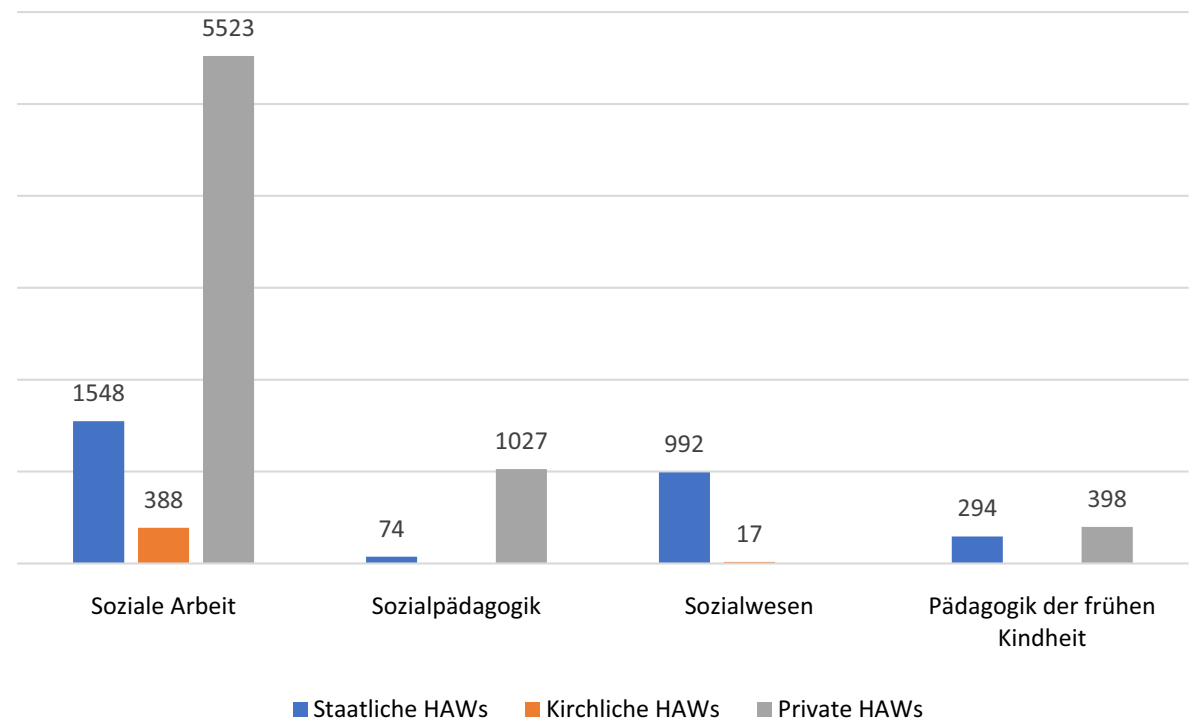

Abb. 2 Entwicklung der Studierendenzahl in den Studiengängen Soziale Arbeit, Sozialpädagogik, Sozialwesen sowie Pädagogik der frühen Kindheit nach Trägerart im Wintersemester 2018/2019 (eigene Darstellung, in absoluten Zahlen)

- Im Fernstudiengang Pädagogik der frühen Kindheit studieren $43 \%$ der Studierenden an einer staatlichen sowie $58 \%$ an einer privaten HAW.

Die Studierendenzahlen entsprechen dabei weitgehend der institutionellen Verteilung: Zehn staatliche, eine kirchliche sowie sieben private HAWs und keine Universitäten sind im Wintersemester 2018/2019 in den genannten Fernstudiengängen aktiv.

Eine beispielhafte Betrachtung der bei der Stiftung Akkreditierungsrat veröffentlichten Modulhandbücher aus dem Bereich Soziale Arbeit, für die anderen durch Destatis ausgewiesenen Studiengänge bedürfte es einer ähnlichen Untersuchung, zeigt dabei folgende Strukturen:

- An privaten HAWs sind die Fern-Bachelorstudiengänge in der Mehrheit mit einem Workload von 180 ECTS ausgewiesen, wobei die Praxisphase für die staatliche Anerkennung mit unterschiedlichen Zeitansätzen (800-960h) ausnahmslos in den Studienverlauf integriert ist.

- Die Regelstudiendauer schwankt in diesen Studiengängen an privaten HAWs zwischen sechs und acht Semestern, wobei staatliche Hochschulen Studiengänge ausschließlich mit einer Regelstudienzeit von acht Semestern anbieten.

- Es werden an privaten HAWs keine Vorgaben - im Gegensatz zu der Mehrzahl der staatlichen HAWs - hinsichtlich relevanter Praxiserfahrung und -dauer vor der Aufnahme des Studiums gemacht. 
- Gleichzeitig differieren die Umrechnungszahlen von einem ECTS zwischen 25 und 30 h. In der Folge ergibt sich eine Differenz zwischen 4500 oder 5400 Studienstunden bei 180 ECTS.

Unberücksichtigt blieben in der Auseinandersetzung mit den Akkreditierungsgutachten, hier bedürfte im Rahmen weiterer Forschung der Einsichtnahme in die Studien- und Prüfungsordnungen aller in der Differenzierung von Destatis genannten Hochschulen, die Anerkennungsmöglichkeiten der einzelnen Hochschulen bspw. mit Blick auf staatlich anerkannte Erzieher*innen oder andere Personengruppen ${ }^{5}$.

\section{Die Ergebnisse der Trendstudie Fernstudium der IUBH}

Die vorliegenden Destatis-Daten zeigen die Bedeutungszunahme des Fernstudiums im Bereich der Sozialen Arbeit. Forschungsergebnisse zu Studierenden und deren Beweggründen sowie Einstellungen zum Fernstudium liegen dabei für den Bereich der Sozialen Arbeit aktuell nicht vor. Aus diesem Grund wird nachfolgend auf die Ergebnisse der Trendstudie Fernstudium der IUBH (Sommerfeld 2019) zurückgegriffen, die seit 2011 alle zwei bis drei Jahre durchgeführt wird. An der Umfrage haben sowohl Personen, die zum Befragungszeitpunkt bereits ein Fernstudium abgeschlossen haben, teilgenommen, als auch Personen, die in ein Fernstudium eingeschrieben waren, einen Fernstudiengang abgebrochen hatten oder Interesse an einem Fernstudium haben. Personen, die angaben sich kein Fernstudium vorstellen zu können, wurden von der Befragung ausgeschlossen. Die Erhebung der Trendstudie Fernstudium wurde im Oktober und November 2018 durchgeführt. Es beteiligten sich rund 5000 Personen, 3675 Personen haben den Fragebogen beendet (Sommerfeld 2019).

Bisher wurde die Trendstudie lediglich kumuliert im Hinblick auf alle befragten Studiengänge veröffentlicht. Für den vorliegenden Beitrag wurden die Befragungsergebnisse einer Sekundäranalyse unterzogen: Ausgewertet wurden nur aktive Studierende, also keine Studieninteressierten oder bereits graduierte Studierende, die aktuell in einem Fernstudiengang einer deutschen Hochschule in der Fächergruppe Soziale Arbeit immatrikuliert sind. Entsprechend ergab sich ein Rücklauf von 304 Fragebögen, was einer Quote von knapp 6\% der erhaltenen Fragebogen entspricht. Aufgrund des hohen Non-response sind die Ergebnisse im Hinblick auf deren Verallgemeinerbarkeit mit Vorsicht zu bewerten. Vor dem professionstheoretischen Hintergrund und der damit verbundenen veränderten Bedeutung der Lizenzierungsphase, erscheint eine Auseinandersetzung mit den Erkenntnissen der Trendstudie, trotz deren Einschränkung in der Repräsentativität, als Möglichkeit einen Eindruck vom aktuellen Stand zu bekommen gleichwohl von hoher Bedeutung.

Die 304 Studierenden der Sozialen Arbeit sind an neun HAWs in der Bundesrepublik immatrikuliert, wovon zwei in staatlicher und sieben in privater Trägerschaft sind. Bei den soziodemografischen Daten der Befragten zeigt sich, dass mit 74\%

\footnotetext{
5 Gleichzeitig wird bei der Durchsicht der Unterlagen der Stiftung Akkreditierungsrat deutlich, dass dort bei Destatis nicht als Fernstudienorte vermerkte weitere Hochschulstandorte bestanden haben oder bestehen müssen. Zur Problematik der Intransparenz bei der statistischen Zählung: Spanu et al. (2020).
} 
die Zahl der Studentinnen in diesem Bereich überwiegt. Damit ist die Anzahl der weiblichen Studierenden im Bereich Soziale Arbeit tendenziell etwas höher als im Vergleich zur Geschlechterverteilung in der Trendstudie allgemein (68\% weiblich). Der Blick in die Anbieterstatistik ,Strukturdaten Distance Learning/Distance Education 2019“ für das Wintersemester 2018/2019 weist einen Frauenanteil von $45 \%$ bei den Studierenden im Bereich Distance Education aus (Fogolin 2019, S. 44) ${ }^{6}$, was den hohen Anteil an weiblichen Studierenden im Bereich der Sozialen Arbeit unterstreicht. Dieser Umstand ist im gesamten Qualifizierungsbereich der Sozialen Arbeit allerdings nicht unüblich: So sind 77,25\% aller Studierenden hier weiblich (Meyer 2020). Die befragten Fernstudierenden der Sozialen Arbeit sind in der Trendstudie zudem mehrheitlich in einem Alter zwischen 25 sowie 35 Jahren $(41,1 \%)^{7}$, was sich so ähnlich auch in den ,Strukturdaten Distance Learning/Distance Education 2019“ zeigt (Fogolin 2019, S. 44) und auch den Erkenntnissen aus der Trendstudie der IUBH - ohne studiengangsspezifische Unterscheidung - tendenziell entspricht (Sommerfeld 2019, S. 16). Der höchste Bildungsabschluss der Fernstudierenden im Bereich Soziale Arbeit variiert zwischen (Fach-)Hochschulreife (60\%), einem bereits abgeschlossenen Hochschulstudium (Bachelor/Master: 11,5\%) und Hauptbzw. Realschulabschluss (17,1\%). Hier überwiegt also - ähnlich bei den Studierenden im Bereich Distance Education (Fogolin 2019, S. 44) - die allgemeine Hochschulzugangsberechtigung. Bei 49,7\% der befragten Studierenden haben die im Haushalt lebenden Personen und/oder die Eltern keinen Hochschulabschluss.

Darüber hinaus wurden im Rahmen der Trendstudie weitere Erkenntnisse zur Lebenssituation der Studierenden in der Sozialen Arbeit generiert. Diese leben in einer festen Partnerschaft $(28,6 \%)$ oder sind verheiratet $(32,9 \%)$. Tendenziell verfügen die befragten Studierenden mit 56,3\% über keine Kinder unter 14 Jahren im eigenen Haushalt, wobei ein knappes Drittel der Studierenden (31,3\%) mit Kindern unter 14 Jahren im Haushalt lebt $^{8}$. Ist dies der Fall, so sind es zumeist eines $(10,9 \%)$ oder zwei $(14,8 \%)$ Kinder. Die befragten Studierenden der Sozialen Arbeit kommen besonders häufig aus den westlichen Bundesländern, wobei sie hier relativ gleichverteilt in größeren (mehr als 50.000 Einwohner*innen), mittleren (zwischen 10.000-50.000) sowie kleineren Kommunen (unter 10.000) leben. Gerade daraus wird deutlich, dass nicht die Erreichbarkeit einer Hochschule entscheidend zu sein scheint, sondern die bewusste Wahl eines Fernstudiengangs im Vordergrund steht. Dies unterstreicht auch die Angabe von 54,6\% der Studierenden, dass persönliche/ private Gründe die Aufnahme eines Präsenzstudiums verhindern. Einmal aufmerksam auf den jeweiligen Studiengang im Modus des Fernstudiums geworden, sind die Flexibilität des Studienmodells (75,3\%), die inhaltliche Ausrichtung des Studiengangs $(63,6 \%)$ sowie der geringe Anteil an Präsenzveranstaltungen $(59,5 \%)$

\footnotetext{
${ }^{6}$ Die Daten beziehen sich nicht speziell auf Studierende der Sozialen Arbeit, schließen Studierende aus den Bereichen Soziale Arbeit, Sozialpädagogik und Sozialwesen aber im Studienbereich Sozialwesen mit ein (Statistisches Bundesamt 2018b, S. 241).

7 Lediglich $26 \%$ der befragten Studierenden sind älter als 36 Jahre und 21,4\% jünger als 25 Jahre.

8 Insgesamt planen etwa $10 \%$ in Elternzeit die Aufnahme von Maßnahmen zur individuellen Professionalisierung (Statista 2019), wobei dies Frauen eher planen als Männer.
} 
relevant, wobei insbesondere die Flexibilität den klassischen Vorteil eines Fernstudiengangs darstellt (Martin 2019).

Darüber hinaus investieren die Studierenden der Sozialen Arbeit viel Zeit in ihr Studium: knapp 50\% studieren in einem Studienmodell mit über $30 \mathrm{~h}$ oder zwischen 15 und $30 \mathrm{~h}(36,8 \%)$ pro Woche. Nach der realen Stundenzahl pro Woche befragt, geben $22,4 \%$ der Studierenden $16-20 \mathrm{~h}$ pro Woche sowie $27 \%$ 11-15 h pro Woche an. 17,4\% arbeiten mehr als $21 \mathrm{~h}$ pro Woche für das Fernstudium und $22,7 \%$ bis zu zehn Stunden. Besonders stark werden dabei der Morgen (35,5\%), der frühe $(45,4 \%)$ sowie der späte Abend $(44,1 \%)$ zum Studium genutzt. Ebenso das Wochenende $(61,2 \%)$ oder Urlaube $(40,5 \%)$. In der Arbeitszeit setzen sich 6,3\% der Studierenden der Sozialen Arbeit mit Inhalten des Fernstudiums auseinander sowie gut $12 \%$ in der Mittagspause. Damit liegen Studierende der Sozialen Arbeit in diesen beiden Tagesstrukturbereichen etwas hinter den Nutzungszahlen aller Studierender (9\% in der Arbeitszeit bzw. 14,2\% in der Mittagspause). Die geringere Integration des Studiums in die Arbeitszeit mag mit darin begründet liegen, dass die Arbeitgeber*innen der befragten Fernstudierenden der Sozialen Arbeit nur in Teilen die individuelle Professionalisierung (vgl. Nittel und Seltrecht 2008) unterstützen: Rückhalt durch den Arbeitgeber erfahren hier nur 24\% der Befragten, $48 \%$ bekommen keine Unterstützung und gut $16 \%$ arbeiten aktuell nicht.

Mit welchem Ziel wird nun ein Fernstudium der Sozialen Arbeit in Angriff genommen? Die Studierenden streben in Summe zumeist $(96,4 \%)$ einen grundständigen Abschluss (Bachelor etc.) an. Insgesamt versprechen sich die Fernstudierenden der Sozialen Arbeit von der Aufnahme des Studiums eine Vertiefung ihres bereits vorhandenen Fachwissens (30,6\%) oder zu 35,2\% eine fachliche Veränderung. Über die Hälfte erwarten die Ermöglichung des eigenen beruflichen Aufstiegs, 39,5\% hoffen auf die Verbesserung von Arbeitsmarktchancen und 35,5\% auf eine Verbesserung der finanziellen Entlohnung. Der höchste Wert mit 53,5\% stellt der Wunsch nach persönlicher Weiterentwicklung dar. Auch wenn der Wunsch nach letzterer besonders hoch erscheint, so ist doch auffällig, dass mit der Wahl eines Fernstudiums eine Verbesserung der beruflichen Situation einhergeht. Bei 53,6\% baut der Fernstudiengang aus dem Bereich Soziale Arbeit entsprechend auch auf der bisherigen beruflichen Laufbahn auf. Vor diesem Hintergrund könnte die Wahl eines Fernstudiums in der Sozialen Arbeit als Instrument der individuellen Professionalisierung (wissenschaftliche berufliche Weiterbildung) für beruflich Tätige in dem Bereich verstanden werden ${ }^{9}$. Dennoch studieren $25 \%$ einen solchen Fernstudiengang als Fachfremde, allerdings mit einer vorhandenen beruflichen Praxis und 10,5\% waren vor Aufnahme des Fernstudiums noch nicht berufstätig. Gerade bei Letzteren stellt

\footnotetext{
9 Robert Pelz und Markus Herklotz (2019) analysieren die Teilnahme von sächsischen Hochschulabsolvent*innen. Sie stellen fest: „Die Mehrheit der [sächsischen, NM und CB] Absolventinnen und Absolventen $(66 \%)$ haben vor der wissenschaftlichen Weiterbildung ein Studium an einer Universität absolviert; etwas mehr als ein Drittel (34\%) an einer Hochschule für angewandte Wissenschaften“ (Pelz und Herklotz 2019, S. 90), wobei ,,[k]umuliert 26\% (...) nach einem bzw. $42 \%$ nach zwei Jahren [nach einem ersten Studienabschluss, NM und CB] in eine Weiterbildung über[gehen]“ (Pelz und Herklotz 2019, S. 91). Weder Bildungsherkunft, Alter, Familienstand oder das Vorhandensein von mindestens einem Kind hat signifikanten Einfluss auf die Teilnahmehäufigkeit an wissenschaftlichen Weiterbildungen (Pelz und Herklotz 2019, S. 92).
} 
sich die Frage warum sich diese Studierenden direkt für ein Fernstudium entschieden haben.

Die Art der didaktischen Aufbereitung stellt für die Studierenden dabei ein wesentliches Entscheidungskriterium für die Aufnahme eines Fernstudiums dar: Eine geringe $(11,5 \%)$ oder sogar überhaupt keine Präsenzphase in den Lehrveranstaltungen $(83,2 \%)$ sind die am stärksten befürworteten Antwortoptionen. Vor diesem Hintergrund überrascht es nicht, dass mehr sozialer Austausch während des Studiums nur von $16,1 \%$ der Studierenden gewünscht wird. Sind sich die Studierenden im Hinblick auf die besonderen didaktischen Optionen des Fernstudiums und deren Nutzen in der Gestaltung des eigenen Lebens bewusst, so wird der Ruf des Fernstudiums bei möglichen Arbeitgeber*innen indifferent eingeschätzt. Während 21,4\% der befragten Studierenden der Sozialen Arbeit glauben, dass ein Fernstudium bei einem Arbeitgeber weniger Wert als ein Präsenzstudium sei - sehen es gut $57 \%$ als gleichwertig an. Dies sehen knapp $52 \%$ aller befragten Fernstudierenden ebenso.

\section{Diskussion und Ausblick}

Die Daten heben die Bedeutung des Fernstudiums für den Lizenzierungsprozess im Bereich der Sozialen Arbeit sowie als Möglichkeit der individuellen Professionalisierung für die Beschäftigten deutlich hervor. Dies hängt jedoch eng mit Fragen der kollektiven Professionalisierung zusammen, denn im Rahmen eines Fernstudiengangs scheint in erster Linie nicht der Erwerb einer rein formalen Berechtigung im Vordergrund zu stehen - der Studiengang baut ja bereits mehrheitlich auf bestehenden beruflichen Kenntnissen auf. Vielmehr scheint es der Wunsch nach persönlicher Weiterentwicklung sowie der Verbesserung der eigenen beruflichen Situation zu sein, welcher die Studierenden antreibt. Insofern stellt das Fernstudium Sozialer Arbeit für eine Mehrheit der befragten Personen gleichsam eine zweite Qualifizierung nach Abschluss einer beruflichen Erstausbildung in diesem Berufssegment und damit im eigentlichen Sinn eine wissenschaftliche Weiterbildung ${ }^{10}$ dar (vgl. Schmid et al. 2019; Wolter und Schäfer 2019). Diese führt gleichzeitig zur formalen Akademisierung bisheriger beruflicher Praxis, womit sich ein wichtiges Kriterium der Professionalisierung Sozialer Arbeit erfüllt (vgl. Meyer 2019a). Dieser Umstand ist aus zwei professionstheoretischen Gründen relevant:

- Professionalisierung beschreibt einen zeitlichen Prozess (vgl. Nittel 2000), der auf zwei verschiedenen Ebenen stattfindet. Sowohl auf der individuellen Ebene, hier verweist Professionalisierung auf den berufsbiografischen Prozess der Qualifizierung und die Erlangung von Professionalität durch eine Fachkraft, als auch auf die kollektive Ebene (vgl. Nittel und Seltrecht 2008; Nittel 2000). Auf dieser stellt Professionalisierung die fachliche Entwicklung und Profilierung sowie die Akademisierung von ganzen Berufsgruppen dar (vgl. Nittel und Seltrecht 2008) In diesem Sinne lässt sich in den Daten eine „Akademisierung auf Umwegen“ er-

10 „Insgesamt ist die unzureichende Datenbasis angesichts der steigenden Bedeutung der wWB [wissenschaftlichen Weiterbildung, NM und CB] erstaunlich“" (Shajek und Winterhager 2019, S. 53). 
kennen, die für den Prozess der Professionalisierung Sozialer Arbeit langfristig positive Konnotationen aufweisen könnte.

- Gleichzeitig wird klar, dass diese „Akademisierung auf Umwegen“ eine besondere Hürde, zeitliche Überschneidungen und durch die fehlende Abstimmung zwischen den Qualifizierungsebenen sowie deren spezifischen Inhalten zu Doppelungen in der Auseinandersetzung mit den jeweiligen Wissensbeständen der Berufsgruppe führen wird. Auch die fehlende Anerkennungspraxis wird hier schlaglichtartig deutlich: An der Tatsache, dass es eine Praxis des Umwegs gibt, wird die Ungleichzeitigkeit der formal im Europäische Qualifikationsrahmen (EQR) gleichgestellten Abschlüsse Fachschulausbildung und Bachelor deutlich. Für die Befragten gibt es „handfeste“ Erwartungen - beruflicher Aufstieg, bessere Entlohnung oder Entfristung (vgl. Meyer und Wahl 2018; Nittel, Schütz und Tippelt 2014) -, die sie zur Akademisierung in ihrem bisherigen Arbeitsfeld antreibt. Gleichwohl wären die Abschlüsse schon längst gleichgestellt und trotzdem scheint es so etwas wie eine unterschiedliche Wertigkeit zu geben. Diese Perspektive lässt wiederrum keineswegs eine positive Entwicklung der Professionalisierung Sozialer Arbeit erkennen.

Insgesamt ist das Geschlechterverhältnis eine wichtige Facette im Fernstudium: Hier sind tendenziell mehr Frauen als im Gesamtverhältnis aller Studierenden eingeschrieben, wobei dies besonders die Fernstudiengänge Sozialwesen und Pädagogik der frühen Kindheit betrifft. Dabei wird ein Fernstudiengang im Bereich der Sozialen Arbeit von Frauen wie Männer besonders in der Altersgruppe der 25 bis 35Jährigen nachgefragt.

Gleichsam sind die Beweggründe für die Aufnahme eines als persönlich herausfordernd wahrgenommenen Fernstudiums (Sommerfeld 2019) bei den befragten Studierenden der Sozialen Arbeit von Bedeutung. Erkenntnisse aus der wissenschaftlichen Weiterbildung zeigen, dass in Gruppen nicht-traditioneller Studierender, also ohne familialen Erfahrungen mit einem Studium, der Wunsch zur Erhöhung des eigenen Wissensstandes offenbar besonders hoch ist (vgl. Gegenfurtner et al. 2019, S. 75). Dies gilt auch für die Befragten der IUBH-Studie (Sommerfeld 2019).

Darüber hinaus fällt auf, dass Sozialpädagogik - im Gegensatz zur Gesamtstudierendenentwicklung (Meyer 2020) - im Fernstudium ähnlich stark wie der Studiengang Sozialwesen nachgefragt wird. Aus der Sonderauswertung der Daten des Bundesamtes für Statistik lässt sich für den Bereich des Fernstudiums außerdem zeigen, dass der Studiengang Pädagogik der frühen Kindheit sein starkes Wachstum eher hinter sich hat und sich nun auf hohem Niveau konsolidiert und fest in der Hochschullandschaft etabliert hat (Fachkräftebarometer Frühe Bildung 2019).

Mit Blick auf die Trägerstruktur wird außerdem ein klarer Unterschied zwischen Präsenzstudium wie dualem Studium deutlich (Meyer 2020): Im prozentualen Verhältnis sind private Träger die Motoren der Entwicklung im Fernstudienbereich, der mehrheitlich an Hochschulen angewandter Wissenschaften vollzogen wird. Einzig der Studiengang Sozialwesen bildet eine Ausnahme. Hier sind staatliche HAWs im Gegensatz zu privaten Hochschulen - besonders stark engagiert. Dies gilt für das im Präsenzstudium ebenso wie für das Fernstudium. 
Abschließend bleibt ein ambivalentes Bild: Einerseits steigen die Zahlen von Fernstudierenden in der Sozialen Arbeit massiv an und gleichzeitig kennen wir die Fernstudierenden in der Sozialen Arbeit bisher so gut wie nicht. Da die Studienmodelle der verschiedenen Hochschulen sehr stark variieren, ist dies allerdings von elementarer Bedeutung. In einer späteren beruflichen Praxis, deren Hauptwerkzeug aber kommunikatives Handeln steht (vgl. von Spiegel 2013), muss diesem Aspekt in der Lizenzierungsphase besonderes Augenmerk geschenkt werden. Bisher haben Profession und Disziplin sich eher Fragen der Entwicklung des dualen Studiums gewidmet (vgl. DGSA 2019; Otto 2018) als die quantitativ bedeutsamere Entwicklung im Bereich des Fernstudiums wahrzunehmen. Die IUBH-Studie zeigt nun durchaus wichtige Einschätzungen der befragten Studierenden: Aus privaten, wie beruflichen Gründen kam ein Präsenzstudium für sie überhaupt nicht in Frage. Insofern entscheiden sich Studierende keineswegs ,aus der Not heraus“ für ein Fernstudium, sondern im Gegenteil bewusst und alternativlos. Aktuell wissen wir über die Studierenden Sozialer Arbeit viel zu wenig, um damit die Präferenzen für die Wahl von Studienmodi (Präsenz, dual, Fern) erklären zu können und entsprechende Förderlinien zur Hochschulforschung fehlen. Vor diesem Hintergrund gilt es zunächst differenziert zu fragen, wer warum welches Studienmodell wählt und wie dieses inhaltlich adäquat auf die spätere berufliche Praxis vorbereiten kann.

Funding Open Access funding enabled and organized by Projekt DEAL.

Open Access Dieser Artikel wird unter der Creative Commons Namensnennung 4.0 International Lizenz veröffentlicht, welche die Nutzung, Vervielfältigung, Bearbeitung, Verbreitung und Wiedergabe in jeglichem Medium und Format erlaubt, sofern Sie den/die ursprünglichen Autor(en) und die Quelle ordnungsgemäß nennen, einen Link zur Creative Commons Lizenz beifügen und angeben, ob Änderungen vorgenommen wurden.

Die in diesem Artikel enthaltenen Bilder und sonstiges Drittmaterial unterliegen ebenfalls der genannten Creative Commons Lizenz, sofern sich aus der Abbildungslegende nichts anderes ergibt. Sofern das betreffende Material nicht unter der genannten Creative Commons Lizenz steht und die betreffende Handlung nicht nach gesetzlichen Vorschriften erlaubt ist, ist für die oben aufgeführten Weiterverwendungen des Materials die Einwilligung des jeweiligen Rechteinhabers einzuholen.

Weitere Details zur Lizenz entnehmen Sie bitte der Lizenzinformation auf http://creativecommons.org/ licenses/by/4.0/deed.de.

\section{Literatur}

Arnold, P., Griesehop, H. R., \& Füssenhäuser, C. (Hrsg.). (2018). Profilierung Sozialer Arbeit online. Innovative Studienformate und Qualifizierungswege. Wiesbaden: Springer VS.

Autorengruppe Fachkräftebarometer (Hrsg.). (2019). Fachkräftebarometer Frühe Bildung 2019. München: DJI.

Deutsche Gesellschaft für Soziale Arbeit (DGSA) (Hrsg.). (2018). Newsletter 1/2018. https://www.dgsa. de/fileadmin/Dokumente/Newsletter/Newsletter_DGSA_1_18-final.pdf. Zugegriffen: 8. Nov. 2018.

Deutsche Gesellschaft für Soziale Arbeit (Hrsg.). (2019). Duale, trägernahe und reguläre Studiengänge Sozialer Arbeit - Qualitätsstandards für eine sich verändernde Hochschullandschaft. Ein Positionspapier des Vorstands der Deutschen Gesellschaft für Soziale Arbeit (DGSA). https://www.dgsa.de/ fileadmin/Dokumente/Aktuelles/DGSA_Stellungnahme_Qualit\%C3\%A4tskriterien_duale_Studieng \%C3\%A4nge_Soziale_Arbeit.pdf. Zugegriffen: 19. Apr. 2019. 
Fogolin, A. (2019). Strukturdaten Distance Learning/Distance Education 2019. Bonn: Bundesinstitut für Berufsbildung.

Gegenfurtner, A., Fisch, K., \& Ebner, C. (2019). Teilnahmemotivation nicht-traditioneller Studierender an wissenschaftlicher Weiterbildung. Beiträge zur Hochschulforschung, 41(4), 58-83.

Hughes, E.C. (1984). The sociological eye. New Brunswick: Transaction Books.

Martin, A. (2019). Das Bedürfnis nach Flexibilität von Fernstudierenden. die hochschullehre, 5(2019), 839-854.

Meyer, N. (2018). Trendstudiengang Soziale Arbeit?! Statistische Daten und ihre professionstheoretische Relevanz. Soziale Passagen. Journal Für Empirie und Theorie Sozialer Arbeit, 10(2), 299-308. https://doi.org/10.1007/s12592-018-0301-x.

Meyer, N. (2019a). Zwischen Aufbruch und Gefährdung. Sozial Extra, 43(5), 335-340. https://doi.org/10. 1007/s12054-019-00219-9.

Meyer, N. (2019b). Neue Unterschiede statt neuer Einigkeit. Sozial Extra, 43(4), 281-286. https://doi.org/ 10.1007/s12054-019-00194-1.

Meyer, N. (2020). Spaltungen im Projekt „Professionalisierung Sozialer Arbeit“: Eine professionstheoretische Deutung am Beispiel der Gesamtstudierendenzahlen. neue praxis, 50(2), 122-140.

Meyer, N., \& Schoneville, H. (2020). Qualifizierung für die Soziale Arbeit. In W. Thole (Hrsg.), Grundriss Soziale Arbeit. Ein einführendes Handbuch. Wiesbaden: Springer VS.

Meyer, N., \& Wahl, J. (2018). Zwischen beruflichem Aufstieg und persönlicher Entwicklung. Sozial Extra, 42(1), 48-51. https://doi.org/10.1007/s12054-017-0113-9.

Nittel, D. (2000). Von der Mission zur Profession? Stand und Perspektiven der Verberuflichung in der Erwachsenenbildung. Bielefeld: Bertelsmann.

Nittel, D., \& Seltrecht, A. (2008). Der Pfad der ,individuellen Professionalisierung“. Ein beitrag zur kritisch-konstruktiven erziehungswissenschaftlichen Berufsgruppenforschung. Zeitschrift für Biographieforschung, Oral History und Lebensverlaufsanalyse, 21(1), 124-145.

Nittel, D., Schütz, J., \& Tippelt, R. (2014). Pädagogische Arbeit im System des lebenslangen Lernens. Ergebnisse komparativer Berufsgruppenforschung. Weinheim: Beltz Juventa.

Otto, H.-U. (2018). Dual - Ende oder Wende des Studiums einer modernen Sozialen Arbeit. neue praxis, 48(3), 297-299.

Pelz, R., \& Herklotz, M. (2019). Wer bildet sich wissenschaftlich weiter? Beiträge zur Hochschulforschung, 41(4), 84-100.

Schmid, C., Maschwitz, A., Wilkesmann, U., \& Nickel, S. (2019). Wissenschaftliche Weiterbildung in Deutschland. Beiträge zur Hochschulforschung, 41(4), 10-35.

Schütze, F. (1992). Sozialarbeit als ,bescheidene“ Profession. In B. Dewe, W. Ferchhoff \& F. Olaf-Radtke (Hrsg.), Erziehen als Profession. Zur Logik professionellen Handelns in pädagogischen Feldern (S. 132-170). Opladen: Leske + Budrich.

Shajek, A., \& Winterhager, N. (2019). Nutzen und Kosten wissenschaftlicher Weiterbildung. Beiträge zur Hochschulforschung, 41(4), 36-56.

Sommerfeld, H. (Hrsg.). (2019). Trendstudie Fernstudium der IUBH. https://www.iubh-fernstudium.de/ wp-content/uploads/IUBH-Trendstudie_Fernstudium-2019_White-Paper_web.pdf. Zugegriffen: 22. Dez. 2019.

Spanu, S., Meyer, N., \& Karsten, M.-E. (2020, i.E.). Qualifizierung für die Qualifizierung zukünftiger Fachkräfte. Beschreibung und Diskussion empirischer Entwicklungen zur Ausbildung von Lehrkräften an Berufsbildenden Schulen mit der Fachrichtung Sozialpädagogik. neue praxis.

v. Spiegel, H. (2013). Methodisches Handeln in der Sozialen Arbeit. Grundlagen und Arbeitshilfen für die Praxis. München: Reinhardt Verlag; UTB.

Statista (Hrsg.) (2019). Was planen Sie während der Elternzeit zu tun?. https://de.statista.com/statistik/ daten/studie/727929/umfrage/geplante-aktivitaeten-in-der-elternzeit-in-deutschland/. Zugegriffen: 22. Dez. 2019.

Statistisches Bundesamt (Destatis) (Hrsg.). (2018a). Bildung und Kultur. Studierende an Hochschulen - Fächersystematik. https://www.destatis.de/DE/Methoden/Klassifikationen/BildungKultur/ StudentenPruefungsstatistik.pdf;jsessionid=C85E62F78FF2F118DB34A36A783C3309. InternetLive1?_blob=publicationFile. Zugegriffen: 7. Jan. 2020.

Statistisches Bundesamt (Destatis) (Hrsg.). (2018b). Bildung und Kultur. Prüfungen an Hochschulen. https://www.destatis.de/DE/Themen/Gesellschaft-Umwelt/Bildung-Forschung-Kultur/Hochschulen/ Publikationen/Downloads-Hochschulen/pruefungen-hochschulen-2110420177004.pdf? blob=publicationFileundv=4. Zugegriffen: 1. Febr. 2020. 
Statistisches Bundesamt (Destatis) (Hrsg.). (2019). Studierende an Hochschulen. Qualitätsbericht. https:// www.destatis.de/DE/Methoden/Qualitaet/Qualitaetsberichte/Bildung/studenten.pdf? blob=publicationFile. Zugegriffen: 15. Okt. 2020.

Thole, W. (2014). Die Soziale Arbeit. Praxis, Theorie, Forschung und Ausbildung. In W. Thole (Hrsg.), Grundriss Soziale Arbeit. Ein einführendes Handbuch (4. Aufl. S. 19-70). Wiesbaden: VS.

Wolter, A., \& Schäfer, E. (2019). Geschichte der wissenschaftlichen Weiterbildung. In W. Jütte \& M. Rohs (Hrsg.), Handbuch Wissenschaftliche Weiterbildung (S. 1-28). Wiesbaden: Springer VS. 